\title{
A comparative study of pancreas transplantation between type 1 and 2 diabetes mellitus
}

\author{
Bor-Shiuan Shyr ${ }^{1}$, Bor-Uei Shyr ${ }^{1,2}$, Shih-Chin Chen $^{1}$, Che-Chuan Loong ${ }^{2}$, Yi-Ming Shyr ${ }^{1}$, Shin-E Wang ${ }^{1}$ \\ ${ }^{1}$ Division of General Surgery, Department of Surgery, Taipei Veterans General Hospital, and National Yang Ming University, Taipei; ${ }^{2}$ Division of \\ Transplant Surgery, Department of Surgery, Taipei Veterans General Hospital, and National Yang Ming University, Taipei \\ Contributions: (I) Conception and design: BS Shyr, BU Shyr, SE Wang; (II) Administrative support: BS Shyr, YM Shyr, SE Wang; (III) Provision \\ of study material or patients: All authors; (IV) Collection and assembly of data: All authors; (V) Data analysis and interpretation: All authors; (VI) \\ Manuscript writing: All authors; (VII) Final approval of manuscript: All authors. \\ Correspondence to: Shin-E Wang, MD. Division of General Surgery, Department of Surgery, Taipei Veterans General Hospital, 201 Section 2 Shipai \\ Road, Taipei 112. Email: sewang0408@gmail.com.
}

Background: Pancreas transplantation remains the best long-term treatment option to achieve physiological euglycemia and insulin independence in patients with labile diabetes mellitus (DM). It is widely accepted as an optimal procedure for type $1 \mathrm{DM}$ (T1DM), but its application in type $2 \mathrm{DM}$ (T2DM) is not unanimously acknowledged.

Methods: In total, 146 diabetes patients undergoing pancreas transplantation were included in this study. Clinical data and outcomes were compared between the T1DM and T2DM groups.

Results: Majority (93\%) of the pancreas transplantations in T2DM were for uremic recipients. Complications occurred in 106 (73\%) patients, including 70 (48\%) with early complications before discharge and 79 (54\%) with late complications during follow-up period. Overall, rejection of pancreas graft occurred in 37 (25\%) patients. Total rejection rate in T2DM recipients was significantly lower than that in T1DM. The short- and long-term outcomes for endocrine function in terms of fasting blood sugar and hemoglobin A1c levels and graft survival rates are comparable between the T2DM and T1DM groups.

Conclusions: T2DM is not inferior to T1DM after pancreas transplantation in terms of surgical risks, immunological and endocrine outcomes, and graft survival rates. Therefore, pancreas transplantation could be an effective option to treat selected uremic T2DM patients without significant insulin resistance.

Keywords: Type 2 diabetes mellitus (T2DM); pancreas; transplantation

Submitted Jun 02, 2019. Accepted for publication Sep 19, 2019.

doi: 10.21037/hbsn-19-422

View this article at: http://dx.doi.org/10.21037/hbsn-19-422

\section{Introduction}

Pancreas transplantation remains the best long-term treatment option to achieve physiological euglycemia and insulin independence in patients with labile diabetes mellitus (DM). It is widely accepted as an optimal procedure for type $1 \mathrm{DM}$ (T1DM), but its application in type $2 \mathrm{DM}(\mathrm{T} 2 \mathrm{DM})$ is not unanimously acknowledged $(1,2)$. Traditionally, pancreas transplantation has been reserved for T1DM patients suffering from uremia, undergoing simultaneous pancreas-kidney (SPK) transplantation, and previously receiving a kidney graft and pancreas after kidney (PAK) transplantation, or those with brittle diabetes and who had undergone pancreas transplant alone (PTA). Historically, pancreas transplantation was considered as a relative, if not absolute, contraindication for T2DM due to its pathophysiology. The reluctance could rely on the pathophysiology of T2DM where insulin resistance on peripheral tissues has been considered as the prevailing disorder, instead of pancreas itself. Therefore, T2DM patients need better peripheral tissue responsiveness to insulin, not extra insulin or pancreas graft. However, the 
distinction between T2DM and T1DM is not always obvious, and many patients may present with overlapping clinical syndromes. Although many criteria, including a family history of diabetes, age of DM onset, body mass index (BMI), human leukocyte antigen (HLA) association, and detectable connecting peptide (C-peptide), have been proposed to differentiate these two types of DM, several patients are still found to categorically overlap. Moreover, older age, associated cardiovascular risks, and advanced secondary diabetic complications might also be suggested as the listed deterrents (3-6).

According to the Scientific Registry of Transplant Recipients (SRTR) report, the proportion of T2DM candidates waiting for SPK transplantation increased from $10.5 \%$ in 2015 to $11.7 \%$ and the proportion of T2DM candidates waiting for PAK transplantation increased from $6.8 \%$ to $8.3 \%$ in 2016 , while the rate of $\mathrm{T} 2 \mathrm{DM}$ candidates waiting for PTA decreased from $3.9 \%$ to $2.9 \%(7,8)$. Although the popularity of pancreas transplantation in T2DM patients remains disproportionately lower than in T1DM, a growing body of evidence has revealed that the endocrine outcome in carefully selected T2DM patients could mirror that of T1DM (3,6,9-13). However, majority of these data regarding pancreas transplantation in T2DM patients are from non-Asian countries, and there is only one Asian study reported by Shin $e t$ al. (10) from Asan Medical Center in Korea.

This study aimed to clarify the justification of pancreas transplantation for T2DM by assessing the surgical risks and comparing the immune and endocrine outcomes and pancreas graft survival rates after pancreas transplantation between T2DM and T1DM patients. We present the following article in accordance with the STROBE reporting checklist (available at https://hbsn.amegroups. com/article/view/10.21037/hbsn-19-422/rc).

\section{Methods}

The authors are accountable for all aspects of the work in ensuring that questions related to the accuracy or integrity of any part of the work are appropriately investigated and resolved. This study was performed in accordance with the Declaration of Helsinki (as revised in 2013). This study was approved by our institutional review board (TPEVGH IRB No.: 2019-06-002AC). The patient consent was waived in view of the retrospective nature of the research and the anonymity of the data.

On September 19, 2003, we initiated our own pancreas transplantation program at Taipei Veterans General Hospital, and from the beginning, we performed pancreas transplantations in T1DM and T2DM candidates. The median waiting period before pancreas transplantation was 10 months for the entire group, 10 months for the T1DM group and 12 months for T2DM group. The median follow-up period after pancreas transplantation was 61 months for the entire group, 65 months for the T1DM group and 48 months for T2DM group. Official indications for pancreas transplantation in Taiwan included the following: (I) T1DM with diabetic complications such as nephropathy, retinopathy, neuropathy, and cardiocerebral vasculopathy; (II) T1DM with frequent lifethreatening hypoglycemia or hyperglycemia; (III) T1DM with severe disability in school learning, working, and living; and (IV) T2DM with kidney disease leading to end-stage renal disease (ESRD) under insulin control with insulin requirement of less than 1.5 units $/ \mathrm{kg} / \mathrm{day}$, which is hopefully to avoid selection of patients with high insulin resistance. T1DM was defined as an early-onset disease with a sudden need for insulin, presence of one or more autoantibodies, and C-peptide negativity. T2DM was defined as a late-onset diabetic disease with detectable fasting C-peptide $>0.8 \mathrm{ng} / \mathrm{mL}$ and without immediate need for insulin for at least 2 years. There were no different criteria for the selection of donors for T1DM and T2DM recipients.

Diabetes patients undergoing pancreas transplantation from September 19, 2003, to November 21, 2018, were included in this study. Data for these patients were prospectively collected and kept in a computer database. This study was approved by our local institutional review board. Clinical data and outcomes including early (before discharge) and late (after discharge) complication rates, surgical mortality, acute and chronic rejections, graft loss, fasting blood sugar, serum hemoglobin A1c (HbA1c), serum C-peptide, and pancreas graft survival were compared between T1DM and T2DM. In this study, any return to insulin use was counted as pancreas graft failure. Diagnosis of the graft rejection was histologically confirmed by needle core-biopsy for each case. Chronic rejection was defined as pancreas graft failure with return to insulin use, and pancreatic graft atrophy by imaging study such as computerized tomography scan and fibrotic change confirmed by graft biopsy.

The primary endpoints of this study were pancreatic, endocrine, and cumulative graft survival outcomes after pancreas transplantation in the T2DM group compared 
with those in the T1DM group. Patient death with functioning graft was regarded as death censor for the cumulative survival outcome. Surgical mortality and technical failure were excluded for survival study.

The secondary endpoints were surgical risks and immunological outcomes in both groups.

Patients with a positive crossmatch against donor cells were excluded for pancreas transplantation. The pancreas grafts were procured in a "no touch" technique en bloc with the duodenum. The spleen was separated from the pancreas before aorta cross-clamping. Histidine-tryptophanketoglutarate solution was used for in situ perfusion. Backtable preparation included removal of the peripancreatic fat and arterial reconstruction using a donor iliac arterial Y-graft. The graft portal vein was anastomosed end-to-side to the recipient's distal vena cava. The superior mesenteric and splenic arteries reconstructed by donor iliac arterial Y-graft at the back-table were anastomosed to the recipient's common iliac or external iliac artery. Exocrine drainage was achieved by enteric drainage with a hand-sewn side-to-side duodenojejunostomy $30-50 \mathrm{~cm}$ beyond the flexure of Treitz ligament using roux-en-Y technique and retroperitoneal placement. No heparin or other anticoagulant was used for every case after pancreas transplantation.

Immunosuppressive therapy included the administration of basiliximab (Simulect, Novartis Pharmaceuticals Corp., East Hanover, NJ, USA), $20 \mathrm{mg}$ given on postoperative days 0 to 4 , or anti-thymocyte globulin (Thymoglobulin ${ }^{\circledR}$; Genzyme, Cambridge, Mass., USA) for high risk of rejection such as positive panel-reactive antibody (PRA) and re-transplantation, $1 \mathrm{mg} / \mathrm{kg}$ daily from postoperative days 1 to 7 , and maintenance therapy included the administration of tacrolimus (Prograf; Astellas Pharma US, Inc., Deerfield, IL, USA), enteric-coated mycophenolic acid (Myfortic; Novartis Pharmaceuticals Corp., East Hanover, NJ, USA), and prednisolone. Prednisolone was tapered and gradually withdrawn 6 months after transplantation. The target trough level for tacrolimus was $8-12 \mathrm{ng} / \mathrm{mL}$ during the first year and $6-8 \mathrm{ng} / \mathrm{mL}$ thereafter.

Statistical analyses were performed using Statistical Product and Service Solutions (SPSS) version 21.0 software (SPSS Inc., IBM, Armonk, NY, USA). All continuous data were presented as median and mean \pm standard deviation $(\mathrm{SD})$, and frequencies were presented when appropriate to the type of data. The mean values of the continuous variables were compared using a two-tailed Student's $t$-test. Nonparametric statistical tests were used if the variables did not follow normal distribution. Categorical variables were presented as numbers and percentages. Categorical variables were compared using Pearson's $\chi^{2}$ test or Fisher's exact test contingency tables. For all analyses, a $\mathrm{P}$ value less than 0.050 was considered statistically significant.

\section{Results}

A total of 146 cases of pancreas transplantation were included in this study, with $115(79 \%)$ for T1DM and 31 (21\%) for T2DM (Table 1). Female gender was predominant in T1DM $(61 \%$ vs. 32\%, $\mathrm{P}=0.005)$. The median age at pancreas transplantation was older for T2DM than for T1DM (40 vs. 32 y/o, $\mathrm{P}<0.001)$. Majority (93\%) of pancreas transplantation for T2DM were for the ESRD groups, including 32\% for SPK transplantation, 19\% for PAK transplantation, and $42 \%$ for pancreas before kidney (PBK) transplantation, compared with T1DM $(45 \%, \mathrm{P}<0.001)$. All of PBK patients had already in uremic status before pancreas transplantation, but they could not get kidney graft at the same time of pancreas transplantation because the waiting lists for pancreas and kidney were separate in our country. The median BMI was higher for T2DM than for T1DM (23 vs. $\left.21 \mathrm{~kg} / \mathrm{m}^{2}, \mathrm{P}=0.024\right)$. There was no significant difference between T1DM and T2DM regarding HLA mismatch, pretransplant panel reactive antibody (PRA), DM duration, and pretransplant insulin requirement.

Table 2 lists the complications and immunological outcomes after pancreas transplantation. Surgical mortality occurred in 3 (2\%) patients, 2 (2\%) with T1DM and $1(3 \%)$ with T2DM. After pancreas transplantation, $106(73 \%)$ patients suffered from complications, including 70 (48\%) with early complications before discharge and 79 (54\%) with late complications during follow-up period. There was no significant difference regarding the complications between the T1DM and T2DM groups. Overall, rejection of pancreas graft occurred in 37 (25\%) patients, including 27 $(19 \%)$ acute rejections and $13(9 \%)$ chronic rejections. Total rejection rate $(10 \%)$ in $\mathrm{T} 2 \mathrm{DM}$ recipients was lower than that $(30 \%)$ in $\mathrm{T} 1 \mathrm{DM}(\mathrm{P}=0.034)$, while acute and chronic rejection rates were not significantly different between these two groups. We also investigated SPK subgroups only, and there was no significant difference regarding the rejection after SPK transplant between T1DM and T2DM, including acute rejection $(19.2 \%$ vs. $10.0 \%, \mathrm{P}=1.000)$, chronic rejection $(11.5 \%$ vs. $10.0 \%, \mathrm{P}=0.392)$, and overall rejection (30.8\% vs. $10.0 \%, \mathrm{P}=0.392)$. Overall, there were 47 (32\%) graft losses, resulting from $6(4 \%)$ acute rejections, 18 (12\%) chronic rejections, 16 (11\%) deaths with functioning graft, 
Table 1 Demographics of patients undergoing pancreas transplantation

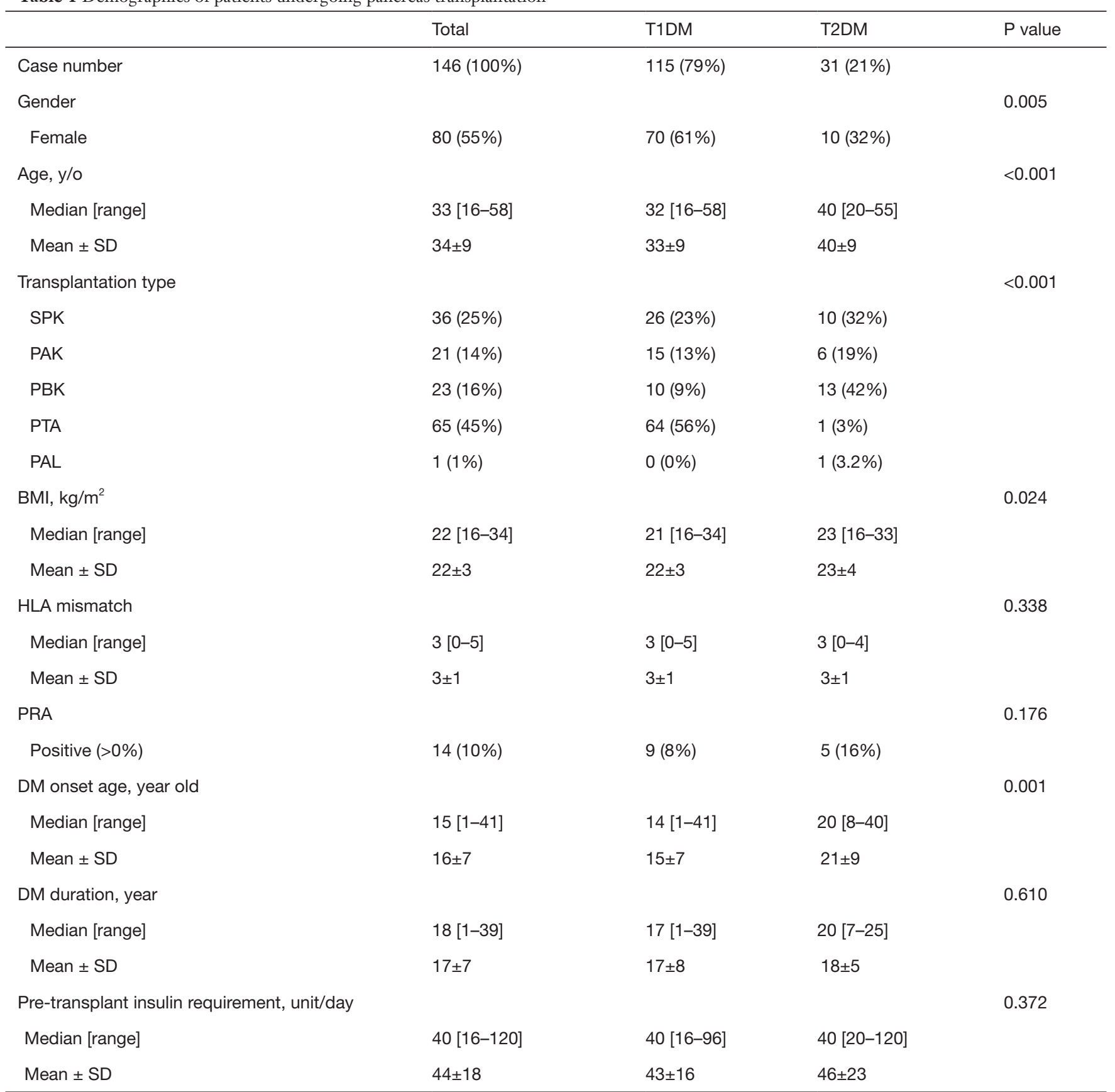

T1DM, type 1 diabetes mellitus; T2DM, type 2 diabetes mellitus; SD, standard deviation; SPK, simultaneous pancreas and kidney; PTA, pancreas transplant alone; PAK, pancreas after kidney; PBK, pancreas before kidney; PAL, pancreas after liver; BMI, body mass index; HLA, human leukocyte antigen; PRA, panel reactive antibody.

$5(3 \%)$ technique failures, and $2(1 \%)$ unknown causes. The graft loss occurred in $35(30 \%)$ T1DM patients and 12 (39\%) T2DM patients $(\mathrm{P}=0.393)$. Technical failure due to graft hemorrhagic pancreatitis occurred in 5 patients $(1$ in T1DM and 4 in T2DM) $(\mathrm{P}=0.007)$.

Endocrine outcomes regarding fasting blood sugar (Figure 1) and serum HbA1c (Figure 2) before and after pancreas transplantation were not significantly different 
Table 2 Complications and immunological outcomes after pancreas transplantation

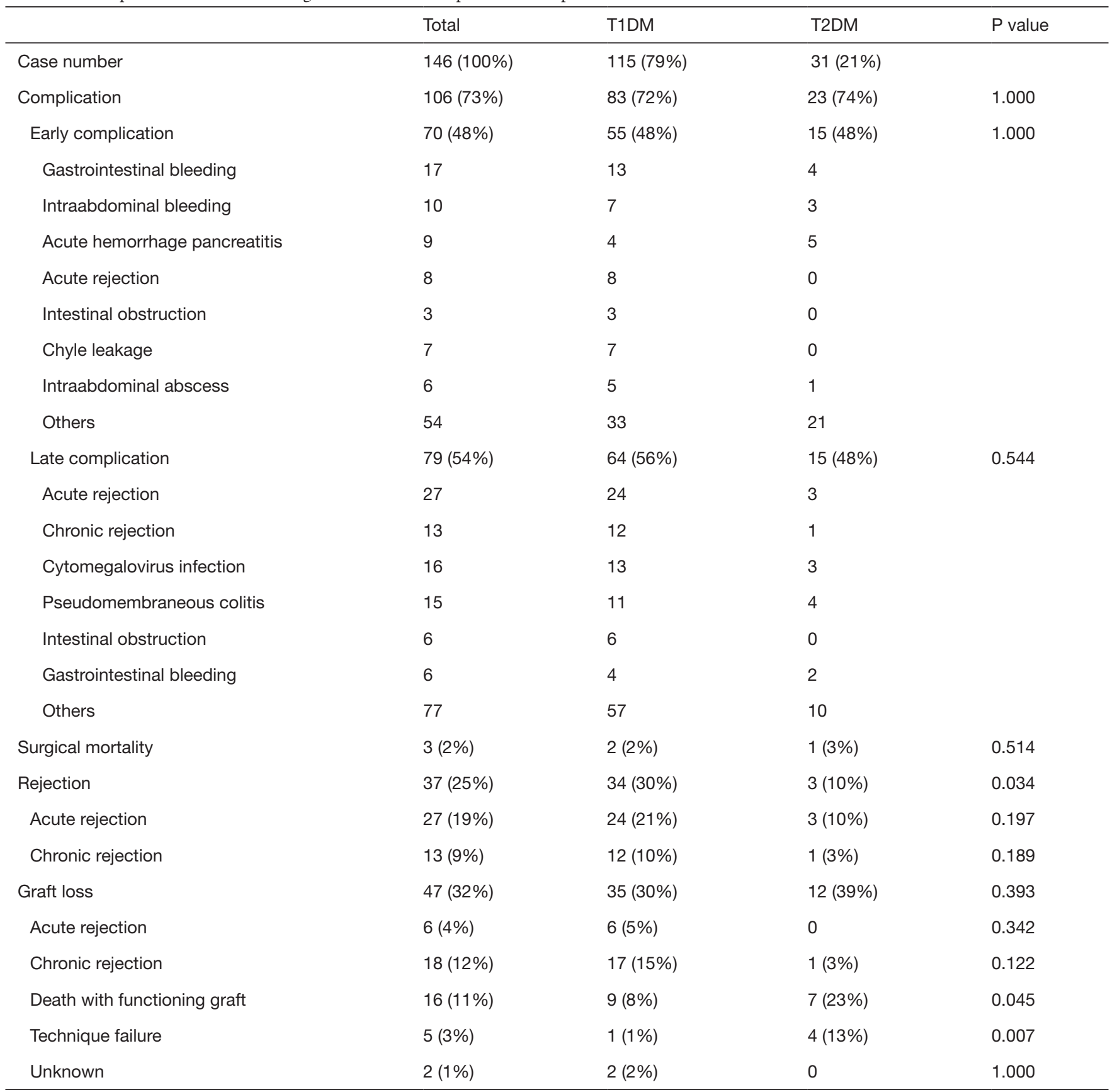

T1DM, type 1 diabetes mellitus; T2DM, type 2 diabetes mellitus; Early complication, complication occurring before discharge; Late complication, complication occurring after discharge.

between the T1DM and T2DM groups. T2DM patients presented significantly higher levels of serum C-peptide either before or after pancreas transplantation compared with T1DM patients (Figure 3). There was always a high peak of serum C-peptide on postoperative day 1 on both T1DM and T2DM patients. For overall patients, 1-, 3-, and 5 -year pancreas graft survival rates were $98.5 \%, 93.2 \%$, and $88.5 \%$, respectively. There was no significant difference 


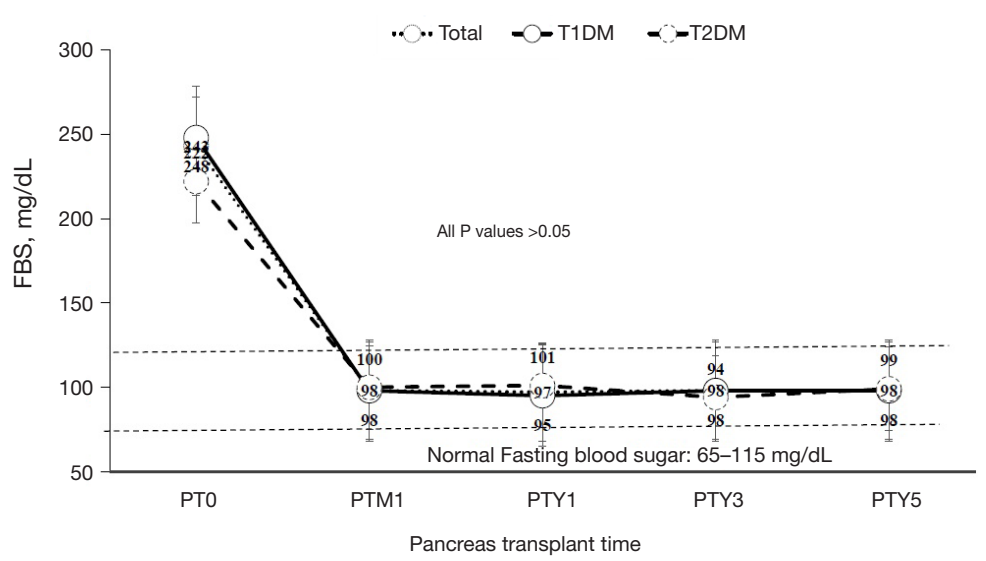

Figure 1 Serum fasting blood sugar (FBS) before pancreas transplant day 0 (PT0), pancreas transplant month 1 (PTM 1), pancreas transplant year 1 (PTY 1), pancreas transplant year 3 (PTY 3), and pancreas transplant year 5 (PTY 5). There is no significant difference regarding FBS between these type 1 diabetes mellitus (T1DM) and type 2 diabetes mellitus (T2DM) before and after pancreas transplant.

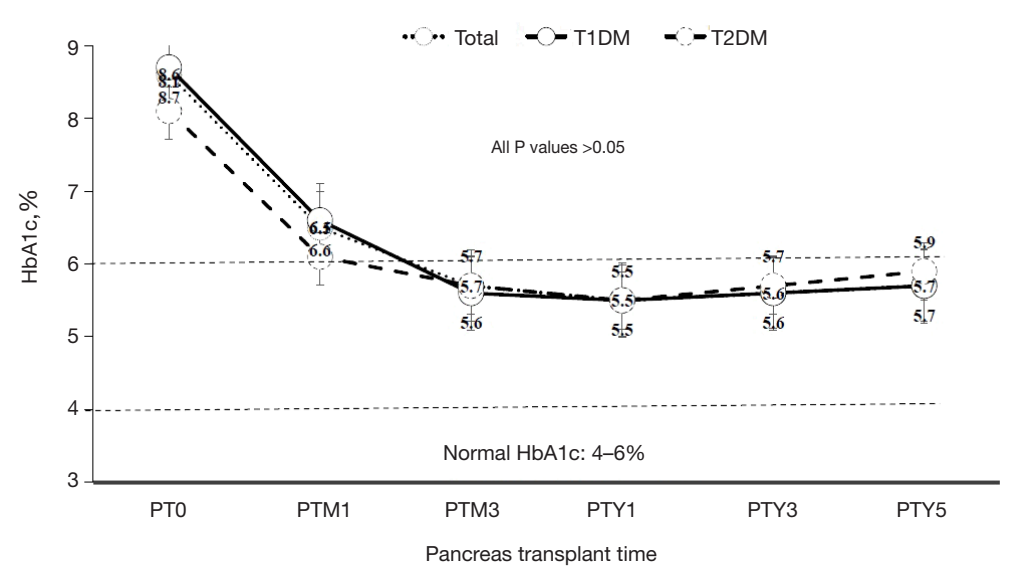

Figure 2 Serum hemoglobulin A1c (HbA1c) before pancreas transplant day 0 (PT0), pancreas transplant month 1 (PTM 1), pancreas transplant month 3 (PTM 3), pancreas transplant year 1 (PTY 1), pancreas transplant year 3 (PTY 3), and pancreas transplant year 5 (PTY 5). There is no significant difference regarding HbA1c between these type 1 diabetes mellitus (T1DM) and type 2 diabetes mellitus (T2DM) before and after pancreas transplant.

regarding the graft survival rates between the T1DM and T2DM groups (Figure 4). For T2DM patients, 1-, 3-, and 5 -year pancreas graft survival rates were all $100 \%$, and for T1DM patients, pancreas graft survival rates were $97.2 \%$, $91.9 \%$, and $86.5 \%$, respectively (Table 3) $(\mathrm{P}=0.138)$. To avoid selection bias, we also investigated SPK groups only, and there was also no significant pancreas graft survival difference between T1DM and T2DM SPK groups (Table 4) ( $\mathrm{P}=0.698)$. Patient survivals were also indicated in Figure 5, and there was no significant difference regarding the patient survival between T1DM and T2DM.

\section{Discussion}

T2DM accounts for up to $90-95 \%$ while T1DM for approximately $5-10 \%$ of all diabetic population. With the increasing epidemic of T2DM, the prevalence of ESRD caused by DM has increased from $15 \%$ in 1980 to $45 \%$ in 2000 and is expected to rise further $(14,15)$. There are multiple options available to control T2DM, including conventional pharmacological intervention (oral hypoglycemic agents and insulin injection), behavioral therapies (mainly diet control, lifestyle changes, and exercise), and bariatric surgery, making it more 


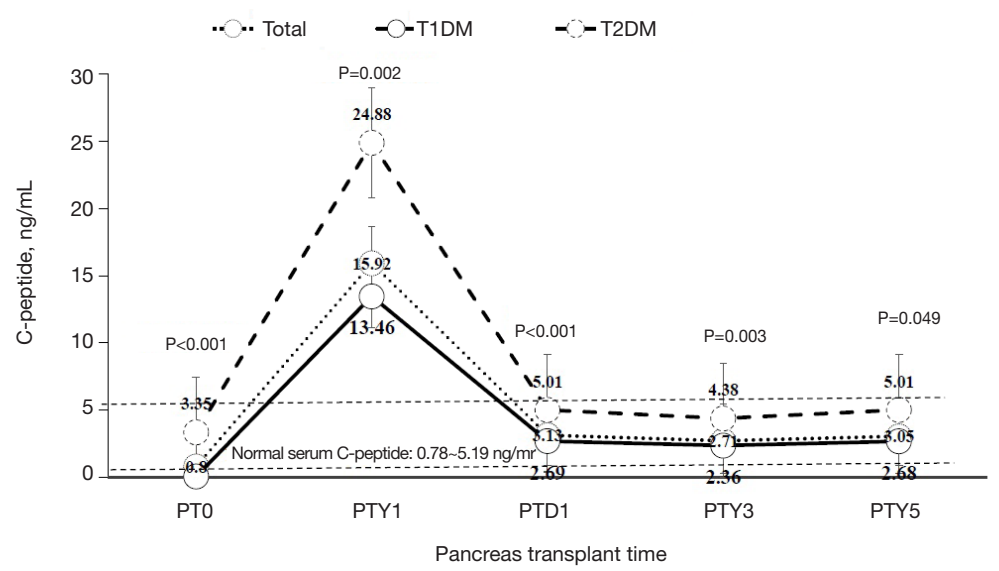

Figure 3 Serum C-peptide before pancreas transplant day 0 (PT0), pancreas transplant day 1 (PTD 1), pancreas transplant year 1 (PTY 1), pancreas transplant year 3 (PTY 3), and pancreas transplant year 5 (PTY 5). Serum C-peptide was significantly higher in type 2 diabetes mellitus (T2DM) patients before and after pancreas transplant. There was usually a high peak of serum C-peptide observed on the day 1 after pancreas transplant in both groups.

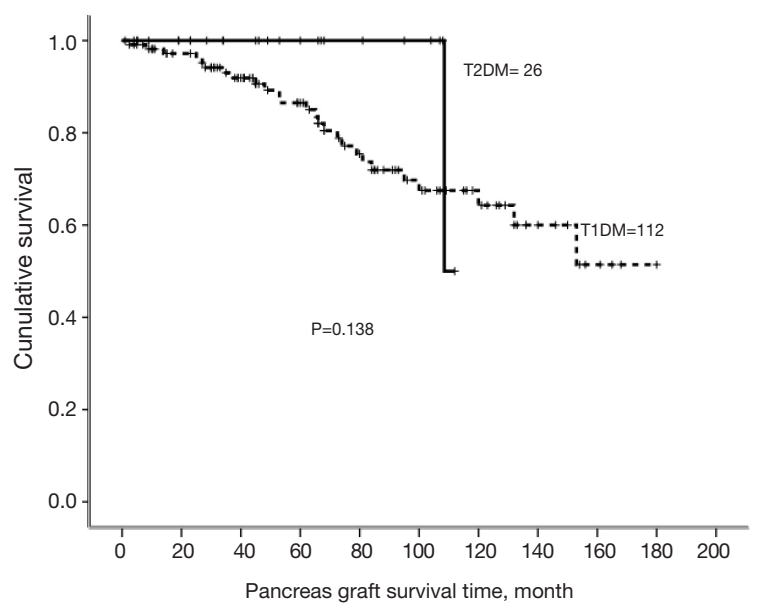

Figure 4 Pancreas graft survival rates after pancreas transplantation for type 1 diabetes mellitus (T1DM) and type 2 diabetes mellitus (T2DM). There is no significant graft survival difference between these two groups.

challenging to decide who would benefit from a pancreas transplantation (16). Moreover, differentiation between T1DM and T2DM based on the presumed mechanisms and clinical presentations is not always distinct because these two groups clinically overlap sometimes $(2,14,17,18)$. Some studies showed that SPK transplantation could be applied in T2DM with acceptable outcomes, but take note that most of the results came from very experienced centers, and these patients were highly selected with particular characteristics such as younger ages, low BMI, and minimal cardiovascular risk (5). Therefore, the best pancreas transplantation option for T2DM is still individualized $(3,14)$.

In our center, pancreas transplantation is considered mainly for those with T2DM, leading to ESRD under insulin control with insulin requirement of less than $1.5 \mathrm{units} / \mathrm{kg} /$ day. There is no significant difference regarding the pre-transplant insulin requirement between T1DM and T2DM because only T2DM without high insulin resistance are included for pancreas transplantation. Thus, no insulin is required after transplantation for these selected T2DM patients during the follow-up period in our series. Under these selection criteria, the rate of pancreas transplantation 
Table 3 Pancreas graft survivals for total patients after pancreas transplantation for T1DM and T2DM

\begin{tabular}{llll}
\hline & Total & T1DM & T2DM \\
\hline Case number & 138 & 112 & 26 \\
Median, month & 61.5 & 65.6 & 47.5 \\
Range, month & $1-180$ & $1-180$ & $4-112$ \\
Mean \pm SD, month & $67.1 \pm 44.4$ & $70.4 \pm 45.6$ & $52.8 \pm 36.2$ \\
1-year survival & $98.5 \%$ & $97.2 \%$ & $100 \%$ \\
3-year survival & $93.2 \%$ & $91.9 \%$ & $100 \%$ \\
5-year survival & $88.5 \%$ & $86.5 \%$ & $100 \%$ \\
\hline
\end{tabular}

T1DM, type 1 diabetes mellitus; T2DM, type 2 diabetes mellitus; SD, standard deviation.

Table 4 Pancreas graft survivals for SPK patients after pancreas transplantation for T1DM and T2DM

\begin{tabular}{llll}
\hline & Overall SPK & T1DM & T2DM \\
\hline Case number & 36 & 26 & 10 \\
Median, month & 105.0 & 112.0 & 88.0 \\
Range, month & $10-180$ & $10-180$ & $34-109$ \\
Mean \pm SD, month & $101.1 \pm 44.7$ & $107.4 \pm 47.3$ & $80.4 \pm 28.7$ \\
1-year survival & $100 \%$ & $100 \%$ & $100 \%$ \\
3-year survival & $96.9 \%$ & $96.0 \%$ & $100 \%$ \\
5-year survival & $93.6 \%$ & $92.0 \%$ & $100 \%$ \\
\hline
\end{tabular}

SPK, simultaneous pancreas and kidney transplantation; T1DM, type 1 diabetes mellitus; T2DM, type 2 diabetes mellitus; SD, standard deviation.

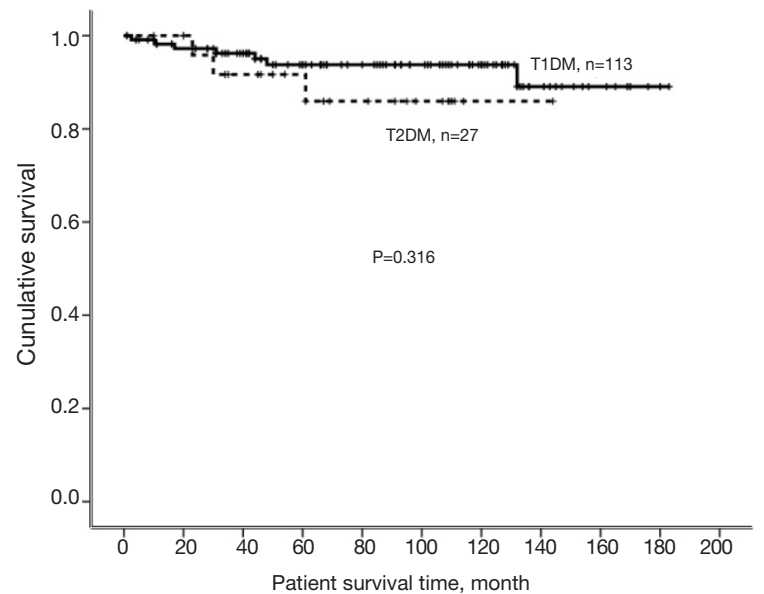

Figure 5 Patient survival rates after pancreas transplantation for type 1 diabetes mellitus (T1DM) and type 2 diabetes mellitus (T2DM). There is no significant patient survival difference between these two groups. 
for T2DM in our center is $21 \%$, which is higher than most of the reports (around 10\%) in the literature $(3,7,8)$, and among them, majority (93\%) are for the ESRD groups including SPK (32\%), PAK (19\%), and PBK (42\%) transplantation. According to the 2015 and 2016 SRTR reported by Kandaswamy et al. $(7,8)$, the number of T2DM recipients undergoing pancreas transplantation increased from $73(8.1 \%)$ in 2015 to 105 (10.8\%) in 2016, and among them, the rate of $\mathrm{T} 2 \mathrm{DM}$ pancreas transplantation increased from $9.7 \%$ to $12.5 \%$ in SPK transplantation, from $2.5 \%$ to $5.4 \%$ in PAK transplantation, and from $0.9 \%$ to $1.9 \%$ in PTA. This substantial increase might indicate a more aggressive approach to perform pancreas transplantation in T2DM. Perhaps, this might be also driven by the substantially shorter waiting time for SPK than for kidney transplant alone (7). The criteria for pancreas transplantation differ from center to center. Some centers require limited insulin resistance (insulin requirement $<1 \mathrm{U} / \mathrm{kg} /$ day), insulin requirement $>5$ years, $\mathrm{BMI}<32 \mathrm{~kg} / \mathrm{m}^{2}$, fasting C-peptide level $<10 \mathrm{ng} / \mathrm{mL}$, presence of glucose hyperlability with complicated diabetes control, age $<60$ years, and minimal cardiovascular comorbidities $(1,4,16,17)$, while other centers use less strict criteria (1). Most centers, including our center, consider pancreas transplantation (SPK or PAK transplantation) mainly for T2DM with ESRD $(1,4,18,19)$. PTA in T2DM remains to be reserved for those with severe metabolic disturbances and incapacitating clinical and emotional problems with exogenous insulin therapy, which would be generally rare among the T2DM patients and should be considered in case-by-case basis (3).

In comparison to T1DM, T2DM recipients in our center are significantly associated with male predominant, older age, higher BMI, and older DM onset age, while pancreas graft loss and surgical risks including complication and mortality remain similar between these two groups. Graft hemorrhagic pancreatitis noted immediately after releasing vascular clamps during operation is the main cause for technical failure in our series. The reason is unknown, but could be due to ischemiareperfusion injury. As to the immunological outcomes, the overall rejection rate is nearly $25 \%$, which is comparable with comparable with current data reported in the literature (7). Total rejection rate is lower in T2DM recipients than in T1DM recipients (10\% vs. $30 \%)$, which could be a reflection of higher proportion of uremic patients in the T2DM group undergoing pancreas transplantation in our series, 93\% (32\% SPK, $19 \%$ PAK and $42 \%$ PBK) vs. $45 \%$.

The short-term (1- and 3-year) and long-term (5-year) outcomes for endocrine function in terms of fasting blood sugar and HbA1c levels and graft survival rates including total patients and only SPK patients are comparable between the T2DM and T2DM groups in this study. The posttransplant levels of fasting blood sugar, HbA1c, and $\mathrm{C}$-peptide remain within the normal range during the follow-up period in both groups, while the posttransplant level of C-peptide is significantly higher in T2DM recipients compared to that in T1DM, which could be a physiological response to higher insulin resistance on peripheral tissues in T2DM. The higher BMI of T2DM could be one of the possible reasons to explain the higher posttransplant level of C-peptide, as high BMI has a risk of T2DM. We also observed that there is always a high peak of serum C-peptide level on day 1 after pancreas transplantation in both the T2DM and T1DM groups, which could be related to ischemia-reperfusion injury, leading to the release of the enzyme stored inside the pancreas graft cells. Previous International Pancreas Transplant Registry analyses have also shown excellent outcomes in both T2DM and T1DM patients who underwent SPK transplantation $(1,18,19)$. Moreover, a plethora of reports in the literature, including case series, single-center reviews, and multiple analyses, have demonstrated noninferior and favorable outcomes of pancreas transplantation in T2DM recipients compared with T1DM recipients $(1-4,6,8-15,18,20-24)$. Therefore, based on our results and evidences in the literature, pancreas transplantation would be justified in selected T2DM patients, especially with ESRD, who have already been under insulin control but without significant insulin resistance.

In conclusion, the posttransplant levels of fasting blood sugar, HbA1c, and C-peptide remain within the normal range during the follow-up period in both groups of this study, and the short-term (1- and 3-year) and long-term (5-year) outcomes for endocrine function in terms of fasting blood sugar and HbAlc levels and graft survival rates are comparable between the T1DM and T2DM groups. Our study shows that T2DM is not inferior to T1DM after pancreas transplantation in terms of surgical risks, immunological and endocrine outcomes, and graft survival rates. Therefore, pancreas transplantation would be justified in selected T2DM patients, especially with ESRD, who have already been under insulin control but without significant insulin resistance.

\section{Acknowledgments}

Funding: This work was financially supported by grants 
from the Taipei Veterans General Hospital (V108C-004 and V108C-003), the Ministry of Science and Technology (MOST 106-2314-B-075-048-MY2), and the Ministry of Health and Welfare (MOHW107-TDU-B-212-114026A).

\section{Footnote}

Reporting Checklist: The authors have completed the STROBE reporting checklist. Available at https://hbsn. amegroups.com/article/view/10.21037/hbsn-19-422/rc

Data Sharing Statement: Available at https://hbsn.amegroups. com/article/view/10.21037/hbsn-19-422/dss

Conflicts of Interest: All authors have completed the ICMJE uniform disclosure form (available at https://hbsn. amegroups.com/article/view/10.21037/hbsn-19-422/coif). The authors have no conflicts of interest to declare.

Etbical Statement: The authors are accountable for all aspects of the work in ensuring that questions related to the accuracy or integrity of any part of the work are appropriately investigated and resolved. This study was performed in accordance with the Declaration of Helsinki (as revised in 2013). This study was approved by our institutional review board (TPEVGH IRB No.: 2019-06-002AC). The patient consent was waived in view of the retrospective nature of the research and the anonymity of the data.

Open Access Statement: This is an Open Access article distributed in accordance with the Creative Commons Attribution-NonCommercial-NoDerivs 4.0 International License (CC BY-NC-ND 4.0), which permits the noncommercial replication and distribution of the article with the strict proviso that no changes or edits are made and the original work is properly cited (including links to both the formal publication through the relevant DOI and the license). See: https://creativecommons.org/licenses/by-nc-nd/4.0/.

\section{References}

1. Gruessner AC, Laftavi MR, Pankewycz O, et al. Simultaneous Pancreas and Kidney Transplantation-Is It a Treatment Option for Patients With Type 2 Diabetes Mellitus? An Analysis of the International Pancreas Transplant Registry. Curr Diab Rep 2017;17:44.

2. Margreiter C, Resch T, Oberhuber R, et al. Combined pancreas-kidney transplantation for patients with end- stage nephropathy caused by type- 2 diabetes mellitus. Transplantation 2013;95:1030-6.

3. Al-Qaoud TM, Odorico JS, Redfield RR 3rd. Pancreas transplantation in type 2 diabetes: expanding the criteria. Curr Opin Organ Transplant 2018;23:454-60.

4. Rohan V, Taber D, Palanisamy A, et al. Impact of Type 1 and Type 2 Diabetes Mellitus on Pancreas Transplant Outcomes. Available online: https://europepmc.org/ article/med/30501589

5. Fourtounas C. Transplant options for patients with type 2 diabetes and chronic kidney disease. World J Transplant 2014;4:102-10.

6. Weems P, Cooper M. Pancreas transplantation in type II diabetes mellitus. World J Transplant 2014;4:216-21.

7. Kandaswamy R, Stock PG, Gustafson SK, et al. OPTN/ SRTR 2016 Annual Data Report: Pancreas. Am J Transplant 2018;18 Suppl 1:114-71.

8. Kandaswamy R, Stock PG, Gustafson SK, et al. OPTN/ SRTR 2015 Annual Data Report: Pancreas. Am J Transplant 2017;17 Suppl 1:117-3.

9. Light J, Tucker M. Simultaneous pancreas kidney transplants in diabetic patients with end-stage renal disease: the 20-yr experience. Clin Transplant 2013;27:E256-63.

10. Shin S, Jung CH, Choi JY, et al. Long-term Metabolic Outcomes of Functioning Pancreas Transplants in Type 2 Diabetic Recipients. Transplantation 2017;101:1254-60.

11. Stratta RJ, Rogers J, Farney AC, et al. Pancreas transplantation in C-peptide positive patients: does "type" of diabetes really matter? J Am Coll Surg 2015;220:716-27.

12. Kaufman DB, Sutherland DE. Simultaneous pancreaskidney transplants are appropriate in insulin-treated candidates with uremia regardless of diabetes type. Clin J Am Soc Nephrol 2011;6:957-9.

13. Gondolesi GE, Aguirre NF, Ramisch DA, et al. Pancreas Transplantation at a Single Latin-American Center; Overall Results with Type 1 and Type 2 Diabetes Mellitus. Transplant Proc 2018;50:1475-81.

14. Cohen DJ, Ratner LE. Type 2 diabetes: the best transplant option is still uncertain. Clin J Am Soc Nephrol 2012;7:530-2.

15. Sampaio MS, Kuo HT, Bunnapradist S. Outcomes of simultaneous pancreas-kidney transplantation in type 2 diabetic recipients. Clin J Am Soc Nephrol 2011;6:1198-206.

16. Ciancio G, Burke GW. Type 2 diabetes: is pancreas transplantation an option? Curr Diab Rep 2014;14:542.

17. Sener A, Cooper M, Bartlett ST. Is there a role for 
pancreas transplantation in type 2 diabetes mellitus? Transplantation 2010;90:121-3.

18. Orlando G, Stratta RJ, Light J. Pancreas transplantation for type 2 diabetes mellitus. Curr Opin Organ Transplant 2011;16:110-5.

19. Gruessner AC, Gruessner RW. Pancreas Transplantation of US and Non-US Cases from 2005 to 2014 as Reported to the United Network for Organ Sharing (UNOS) and the International Pancreas Transplant Registry (IPTR). RDS 2016;13:35-58.

20. Singh RP, Rogers J, Farney AC, et al. Do pretransplant C-peptide levels influence outcomes in simultaneous kidney-pancreas transplantation? Transplant Proc 2008;40:510-2.

21. Chakkera HA, Bodner JK, Heilman RL, et al. Outcomes

Cite this article as: Shyr BS, Shyr BU, Chen SC, Loong CC, Shyr YM, Wang SE. A comparative study of pancreas transplantation between type 1 and 2 diabetes mellitus. HepatoBiliary Surg Nutr 2021;10(4):443-453. doi: 10.21037/ hbsn-19-422 after simultaneous pancreas and kidney transplantation and the discriminative ability of the C-peptide measurement pretransplant among type 1 and type 2 diabetes mellitus. Transplant Proc 2010;42:2650-2.

22. Gruessner AC, Gruessner RWG. Pancreas Transplantation for Patients with Type 1 and Type 2 Diabetes Mellitus in the United States: A Registry Report. Gastroenterol Clin North Am 2018;47:417-41.

23. Nath DS, Gruessner AC, Kandaswamy R, et al. Outcomes of pancreas transplants for patients with type 2 diabetes mellitus. Clin Transplant 2005;19:792-7.

24. Light JA, Barhyte DY. Simultaneous pancreas-kidney transplants in type I and type II diabetic patients with endstage renal disease similar 10-year outcomes. Transplant Proc 2005;37:1283-4. 